\title{
Frequency of Cervical Nodal Metastasis in Early- Stage Squamous Cell Carcinoma of the Tongue
}

\author{
Sadaf Qadeer Ahmed ${ }^{1}$ \\ Montasir Junaid ${ }^{2}$ \\ Sohail Halim 6 \\ ${ }^{1}$ Department of Otorhinolaryngology, Sir Syed College of Medical \\ Sciences for Girls, Karachi, Pakistan \\ ${ }^{2}$ Department of Otorhinolaryngology, Armed Forces Hospitals \\ Southern Region, Khamis Mushayt, Saudi Arabia \\ ${ }^{3}$ Department of Otorhinolaryngology, Aga Khan University, \\ Karachi, Pakistan \\ ${ }^{4}$ Department of Otorhinolaryngology, Manchester Royal Infirmary, \\ Manchester, United Kingdom of Great Britain and Northern Ireland \\ ${ }^{5}$ Department of Medicine, Shifa College of Medicine, \\ Islamabad, Pakistan \\ ${ }^{6}$ Department of Pathology, Aga Khan University, Karachi, Pakistan
}

Sohail Awan ${ }^{3}$

Maliha Kazi ${ }^{4}$

Hareem Usman Khan ${ }^{5}$

Address for correspondence Sadaf Qadeer Ahmed, Department of Otorhinolaryngology, Sir Syed College of Medical Sciences for Girls, Korangi Road, Karachi, Karachi 74800, Pakistan

(e-mail: sadaf.qkhan@gmail.com; sadaf_q1@hotmail.com).

Int Arch Otorhinolaryngol 2018;22:136-140.

\begin{abstract}
Introduction Oral cavity carcinoma is an aggressive tumor, with the tongue being one of the most common subsites of involvement. Surgery is a gold standard method of dealing with advanced-stage tumors. However, for early-stage carcinomas of the tongue, the management remains controversial. Several studies have indicated that early-stage cancers have a high chance of occult cervical node metastasis, which, if left untreated, can greatly affect the prognosis. Certain parameters can help identify patients with occult cervical node metastases, and can avoid unnecessary neck dissection in node negative patients. Tumor thickness is one such objective parameter. Objective To estimate the frequency of cervical lymph node metastasis in patients with early-stage, node-negative $\left(\mathrm{N}_{0}\right)$ squamous cell carcinoma of the tongue.

Methods In-patient hospital data was reviewed from January 2013 until March 2014, and 78 patients who underwent primary resection of the tumor and neck dissection for biopsy-proven, early stage squamous cell carcinoma of the tongue were included. Data such as tumor thickness, tumor differentiation and presence of occult nodal metastasis in the surgical specimen were gathered from the histopathology reports. The frequency of subclinical cervical lymph node metastasis in patients with early-stage squamous cell carcinoma of the tongue was estimated.

Keywords

- squamous cell carcinoma

- tongue neoplasms

- neck dissection

Results A total of $69 \%$ of the patients with tumor thicknesses $>5 \mathrm{~mm}$ had tumor metastases in the neck nodes, while $100 \%$ of the patients with tumor thicknesses $<5$ $\mathrm{mm}$ had no neck nodal metastasis.

Conclusion A tumor thickness $>5 \mathrm{~mm}$ is significantly associated with subclinical metastasis, and prophylactic neck dissection is warranted in such cases.
\end{abstract}

received

November 7, 2016

accepted

April 1, 2017

published online

June 6, 2017
DOI https://doi.org/

10.1055/s-0037-1603626.

ISSN 1809-9777.
Copyright $\odot 2018$ by Thieme Revinter

Publicações Ltda, Rio de Janeiro, Brazil
License terms

(c) (i) $\ominus$ (\$) 


\section{Introduction}

Oral cancer is an important health issue. With a global incidence of 10 million cases in 2000, it is among the most frequent head and neck malignancies; and this figure is further expected to rise to $\sim 15$ million by $2020 .{ }^{1}$ Among the subsites of the oral cavity, the tongue is one of the most common sites for the occurrence of squamous cell carcinoma. ${ }^{2}$

Squamous cell carcinoma of the tongue has high potential for local invasion, and great propensity for metastasis in the cervical lymph nodes. ${ }^{3}$ Thus, cervical lymph node metastasis is considered an important prognostic factor for squamous cell carcinoma of the tongue., ${ }^{4,5}$

The management of cervical nodal metastasis has never been a problem in late-stage (III and IV) of squamous cell carcinomas of the tongue, because the nodes are usually palpable upon clinical examination or evident upon radiological examination. In such cases, definite elective neck dissection is recommended in the literature. ${ }^{6}$

In the early stages of squamous cell carcinoma of the tongue (stages I and II), the cervical lymph nodes are usually neither clinically palpable, nor seen upon radiologic examination (node-negative $\left[\mathrm{N}_{0}\right]$ neck). Still, these early-stage tongue carcinomas may harbor micro-metastasis in the lymph nodes. This incidence is between $27-42 \%$, as reported in the literature. ${ }^{6,7}$ However, there is a lot of debate concerning the best way to approach such a neck disease in early-stage tongue carcinoma (T1 and T2). ${ }^{6}$ Should elective neck dissection be performed, or is a "wait and see" policy safe and adequate in these cases?

The benefits of elective neck dissection are: 1 ) it provides pathologic information on the status of the neck nodes, thus helping to determine the need for additional therapy; and 2) it can also remove undetectable cancer cells lodged in the lymph vessels and nodes (micro-metastasis). ${ }^{8}$ However, the drawbacks are: 1) many patients may not need such treatment, as they might not have micro-metastasis, and would undergo unnecessary neck dissection; and 2) neck dissection has an associated morbidity, and may remove or destroy a natural barrier against cancer spread in these patients. ${ }^{9}$

This controversy in the management of $\mathrm{N}_{0}$ necks ${ }^{10,11}$ has led clinicians to look for reliable preoperative parameters that predict neck nodal micro-metastasis, ${ }^{5,12}$ which, in turn, will help avoid unnecessary neck dissections and save patients from undue financial constraints and morbidity at the same time. Such parameters include grade, size and thickness of the tumor.

Recently, microarray analysis on oral squamous cell carcinoma (OSCC) related genes by Masaki et $\mathrm{al}^{13}$ revealed that some genes are overexpressed in well-differentiated OSCCs with lymph node metastases when compared with those without nodal disease. These OSCCs with nodal diseases exhibited increased levels of MMP-1, MMP-3, uPA, integrin$\alpha 3$, paxillin, tenascin C and IL-6 transcripts, and could be used as potential biomarkers for lymph node metastasis in the future. This technique is unavailable in Third World countries; still, it needs randomized trials with larger cohorts to be proven beneficial and effective.
Given the high incidence of squamous cell carcinoma of the tongue in our part of the world coupled with the high morbidity associated with unnecessary neck dissections in $\mathrm{N}_{0}$ necks, our aim was to determine the frequency of cervical lymph node metastasis in early-stage, $\mathrm{N}_{0}$ tumors. We also looked for parameters such as tumor thickness to help us make the decision for neck dissection in those patients.

\section{Material and Methods}

From January 2013 to March 2014, a total of 78 patients were treated for biopsy-proven squamous cell carcinoma of the tongue at our center. Out of the total, 78 patients with earlystage (T1-T2) squamous cell carcinomas of the tongue, without evidence of neck nodal metastasis upon palpation or computed tomography (CT) and magnetic resonance imaging (MRI) scans of the neck were included in this cross sectional study, regardless of age and gender. All patients underwent a routine tumor resection followed by a neck dissection, while patients with previous history of treatment for squamous cell carcinoma of the oral cavity by any modality (surgical resection, chemotherapy or radiation) were excluded. To reduce the bias, patients with advanced-stage (T3 and T4) carcinoma of the tongue were also excluded.

\section{Data Collection}

All the patients who fulfilled the inclusion criteria were enrolled in the study through the online in-patient hospital database. All the necessary parameters (age, gender, and tumor stage) were recorded in the proforma (-supplementary material [online only]) by the principal investigator, which also included the tumor dimension in terms of thickness $(<5 \mathrm{~mm}$ or $>5 \mathrm{~mm})$ and the presence of metastatic nodes from the pathology specimen report.

Excision of the tongue tumor and neck dissection were performed in the usual manner, under general anesthesia by an experienced surgeon with the assistance of senior residents. As per routine and under institutional policy, all the primary tumors and the neck dissection levels are always sent in separate containers to the histopathology department. A single, experienced and dedicated histopathologist performs the routine step, serial sectioning, and measures the tumor thickness in all primary specimens in millimeters, and checks the neck dissection specimens for the presence of metastasis.

\section{Data Analysis}

Data was analyzed using the Statistical Package for the Social Sciences (SPSS, IBM Corp., Armonk, NY, US), version 20. The continuous variables, such as age, were analyzed as means with standard deviations. The categorical data, that is, sex, and the grade, stage, and thickness ( $\leq 5 \mathrm{~mm}$ or $>5 \mathrm{~mm}$ ) of the tumor, as well as the presence of lymph node metastasis, were analyzed as frequencies (\%). A stratified analysis of the effect modifiers, that is, tumor thickness, T-stage and grading was also performed, as well as a post-stratification chisquare test. Values of $p<0.05$ were deemed significant. 
Table 1 Association of neck lymph node metastasis with tumor thickness

\begin{tabular}{|l|l|l|l|}
\hline \multirow{2}{*}{ Tumor thickness } & \multicolumn{2}{|l|}{$\begin{array}{l}\text { Neck lymph node } \\
\text { metastasis }(\boldsymbol{n}=78)\end{array}$} & \multirow{2}{*}{$\boldsymbol{p}$-value } \\
\cline { 2 - 3 } & Yes & No & \\
\hline$\leq 5 \mathrm{~mm}$ & $0(0 \%)$ & $49(100 \%)$ & \multirow{2}{*}{0.0001} \\
\hline$>5 \mathrm{~mm}$ & $20(69.0 \%)$ & $9(31.0 \%)$ & \\
\hline Total & $20(26.6 \%)$ & $58(74.4 \%)$ & \\
\hline
\end{tabular}

\section{Results}

Among the 78 patients identified, the mean age was 47.5 years, with a standard deviation of 7.5 years. Out of the 78 patients, 61 (78.2\%) were male, and 17 (21.8\%) were female.

Among the 78 cases, there were 23 (29.5\%) well-differentiated tumors, while 39 (50\%) were moderately-differentiated tumors, and 16 (20.5\%) were poorly-differentiated tumors. In this regard, the most common grade was moderately differentiated carcinoma. With respect to the stage of the disease, 25 (32.1\%) tumors were categorized as T1, while 53 (67.9\%) were categorized as T2 lesions. A total of $49(62.8 \%)$ cases had a tumor thickness $\leq 5 \mathrm{~mm}$, while 29 (37.2\%) had a tumor thickness $>5 \mathrm{~mm}$. Neck nodal metastases from the tumor were found in 20 patients ( $25.6 \%)$, while 58 patients $(74.4 \%)$ were found to have no disease in the neck, that is, no neck nodal metastasis. Hence, overall, the number of patients without neck nodal metastasis was greater (74.4\%) then the number of patients with neck nodal metastasis (25.6\%) (- Table 1). Interestingly, neck nodal metastasis was significantly correlated to tumor thickness $>5 \mathrm{~mm}(p<0.0001)$.

Among the patients with T1 lesions, only $2(8 \%)$ had tumor thicknesses $>5 \mathrm{~mm}$, while the remaining 23 (92\%) patients had tumor thicknesses $\leq 5 \mathrm{~mm}$. In patients with T2 lesions there were 26 (49.1\%) with tumor thicknesses $\leq 5 \mathrm{~mm}$, and 27 (50.9\%) with tumor thicknesses $>5 \mathrm{~mm}$ ( - Table 2). Hence, a greater number of patients with T2 lesions had tumor thicknesses $>5 \mathrm{~mm}$, and the association was found to be statistically significant $(p<0.0001)$.

The patients with T1 lesions $(n=25)$ showed no neck nodal metastasis. Among those with T2 lesions $(n=53), 20$ (37.7\%) patients were found to have neck nodal metastasis, and $33(63.3 \%)$ patients were found to have no metastasis in their necks (-Table 2 ). The association between the T-stage of the disease and neck nodal metastasis was also statistically significant $(p<0.0001)$.
Table 3 Association of grade of tumor with neck lymph node metastasis

\begin{tabular}{|l|l|l|l|}
\hline \multirow{2}{*}{ Grade of tumor } & \multicolumn{2}{|l|}{$\begin{array}{l}\text { Neck lymph node } \\
\text { metastasis }\end{array}$} & \multirow{2}{*}{-value } \\
\cline { 2 - 3 } & Yes & No & \\
\hline Well-differentiated & $1(4.3 \%)$ & $22(95.7 \%)$ & \multirow{2}{*}{0.001} \\
\cline { 1 - 3 } $\begin{array}{l}\text { Moderately- } \\
\text { differentiated }\end{array}$ & $10(25.6 \%)$ & $29(74.4 \%)$ & \\
\hline Poorly-differentiated & $9(56.2 \%)$ & $7(43.8 \%)$ & \\
\hline Total & $20(25.6 \%)$ & $58(74.4 \%)$ & \\
\hline
\end{tabular}

Among the cases of well-differentiated tumors, only 1 (4.3\%) patient showed neck nodes that were positive, while the remaining 22 (95.7\%) patients had no neck nodal metastasis. In the cases of moderately-differentiated tumors ( $n=39), 10$ (25.6\%) patients had positive neck nodes, and 29 (74.4\%) patients had no positive neck nodes. Among the cases of poorly-differentiated tumors $(n=16), 9(56.2 \%)$ patients had neck nodal metastases, while 7 (43.8\%) patients were tumorfree. We observed that poorly-differentiated tumors had the highest incidence of neck nodal metastasis (56.2\%), followed by moderately-differentiated tumors (25.6\%), while well-differentiated tumors were the least affected (4.3\%) by neck nodal metastasis (-Table $\mathbf{3}$ ). This association between the grade of the tumor and the neck nodal metastasis was also statistically significant $(p=0.001)$.

\section{Discussion}

Squamous cell carcinoma of the head and neck has been known to have an unpredictable clinical behavior. This feature has led clinicians to seek factors that may help identify early cervical metastases, since harboring a metastasis in the neck significantly reduces the prognosis. ${ }^{14}$

These factors help clinicians avoid unnecessary neck dissections and predict the nature of the head and neck squamous cell disease. Of all the parameters, tumor thickness in particular is a quantifiable measurement, likely to be precise and accurate, while other parameters (such TNM, extra capsular spread) are not only qualitative (subjective bias), but are also liable to vary from one histological section to the other. ${ }^{8}$ Tumor thickness has been reported to be a significant prognostic factor for the occurrence of subclinical and clinical metastases in the literature. ${ }^{14-18}$ Similarly, our results also showed a strong correlation between tumor thickness and neck metastasis.

Table 2 Tumor thickness with different parameters (number of patients, T-stage with positive neck nodes)

\begin{tabular}{|l|l|l|l|l|l|}
\hline Tumor thickness & Percentage of patients (\%) & $\begin{array}{l}\text { T1 } \\
\text { stage (\%) }\end{array}$ & $\begin{array}{l}\text { T2 } \\
\text { stage (\%) }\end{array}$ & $\begin{array}{l}\text { Node positive } \\
\text { T1 lesion (\%) } \\
\mathrm{N}=25 \text { patients }\end{array}$ & $\begin{array}{l}\text { Node positive } \\
\text { T2 lesion (\%) } \\
\mathrm{N}=53 \text { patients }\end{array}$ \\
\hline$<5 \mathrm{~mm}$ & 62.80 & 92.00 & 49.10 & 0 & 0 \\
\hline$>5 \mathrm{~mm}$ & 37.20 & 8.00 & 50.90 & 0 & $37.73^{*}$ \\
\hline
\end{tabular}

Note: ${ }^{*} p$-value $<0.0001$ 
Martinez-Gimeno et al studied the risk of nodal metastasis in 126 patients with oral cavity and oropharyngeal carcinomas. The risk of nodal metastasis was found to be correlated with 7 parameters, including T-stage, intravascular invasion, tumor grade, tumor thickness, tumor host interphase, inflammatory infiltrate, and perineural spread. According to the scoring system used in the study, only tumor thickness was found to be an independent factor in the prediction of subclinical neck node metastases. ${ }^{19}$

Nodal recurrences are the main causes of treatment failure of stage I and II tongue carcinomas. A better prognostic evaluation system is therefore necessary to guide the clinical management, particularly in the consideration of elective neck dissection for subclinical nodal metastasis. The management of the $\mathrm{N}_{0}$ neck in early-stage squamous cell carcinoma of the tongue has been the subject of heated debate over the years.

Keski-Santti et al observed that, for stage I and II oral tongue squamous cell carcinomas, there were significantly fewer regional recurrences in patients with elective neck dissection. They concluded that the risk of occult cervical metastasis was high in patients with early tongue tumors, and carefully selected only patients that could be left without prophylactic neck treatment. ${ }^{20}$ In our study, we included the patients with early-stage tongue carcinoma (T1 and T2 lesions). A total of $67.9 \%$ of the patients in our study population had T2-stage diseases.

Since early-stage tongue carcinoma is a locoregional disease, recurrence and distant metastasis are rare; the rate of overall occult neck nodal metastasis in our patients was of $25 \%$, which is almost similar to that reported by Pimenta Amaral et $\mathrm{al}^{21}$ (23\%) and Kligerman et $\mathrm{al}^{22}$ (21\%).

It is generally recommended that, for squamous cell carcinoma of the upper aerodigestive tract, the clinically $\mathrm{N}_{0}$ neck should be treated when the incidence of occult metastasis is greater than $20 \%{ }^{23}$

Grading tumors based on differentiation (as well-, moderately-, and poorly-differentiated) has been routinely used in the past, with increasing rates of regional metastasis as the grade of the tumor increases from well- to poorly-differentiated. Similar results were observed in a study by Yuen et $\mathrm{al}^{24}$ on laryngeal carcinoma with subclinical metastasis. However, with early tongue lesions (T1 and T2), no correlation was observed by Byer et al. ${ }^{25}$ In our study of early tongue carcinoma, we observed that patients with poorly-differentiated squamous cell carcinoma had more neck node metastases (56.2\%), followed by those with moderately-differentiated squamous cell carcinoma (25.6\%).

Our study showed a tumor thickness $>5 \mathrm{~mm}$ to be significantly associated with neck nodal metastasis. A similar study by Asakage et al on early tongue carcinomas after multivariate analysis demonstrated that a tumor thickness $>4 \mathrm{~mm}$ was one of the most important and powerful factors to predict subclinical neck nodal metastasis. ${ }^{14}$ Po Wing Yuen et al showed that a tumor thickness $<3 \mathrm{~mm}$ was significant in patients who had no neck metastasis. ${ }^{7}$ Tumor thicknesses of 3 to $9 \mathrm{~mm}$ and $>9 \mathrm{~mm}$ were associated with a high rate of subclinical neck nodal metastasis.
Keski-Santti et $\mathrm{al}^{20}$ observed that as the tumor thickness increases over $2 \mathrm{~mm}$. The rate of occult/subclinical metastases in early tongue carcinomas increases from 36 to $39 \%, 41 \%$, and $46 \%$, in $>3 \mathrm{~mm},>4 \mathrm{~mm},>5 \mathrm{~mm}$ and $>6 \mathrm{~mm}$ respectively. Their inference was that after $5 \mathrm{~mm}$, the rate of subclinical metastasis is significantly increased. In our study, out of 78 patients, there was no neck node metastasis in patients with tumor thicknesses $\leq 5 \mathrm{~mm}$, while among the patients with tumor thicknesses $>5 \mathrm{~mm}(n=29), 20$ (69.0\%) had neck nodal metastasis.

Similarly, Fukano et al demonstrated that, among 34 patients, cervical metastasis increased from $5.9 \%$ for tongue carcinomas $<5 \mathrm{~mm}$ thick to $64.7 \%$ for tongue carcinomas $>5 \mathrm{~mm}$ thick. ${ }^{15}$ With a slight variation, Fakih et al noted that, among T1 and T2 squamous cell carcinomas of the tongue, a thickness $>4 \mathrm{~mm}$ is associated with a greater risk of neck relapse. ${ }^{17}$ Another study noted that the rate of occult cervical metastases increased from 7 to $30 \%$ when the thickness of the tumor was $\geq 4 \mathrm{~mm}^{22}$

Similarly, T-staging also showed that there was a high number (37.7\%) of neck nodal metastases in T2 tumors, further emphasizing the importance of elective neck treatment in advanced-stage tongue squamous cell carcinomas. Our results correspond to those of similar studies, ${ }^{16,20,25}$ which showed a high chance of cervical node metastasis with an increase in T-stage. However, Keski-Santti et al observed 2 patients with T1-tumors with superficial depths of invasion $(1.3 \mathrm{~mm}$ and $1.5 \mathrm{~mm}$ ) who had subclinical metastases, and suggested that all patients with early tongue squamous cell carcinomas should be treated with suspicion of harboring neck metastases. $^{20}$ In our study, 2 patients with T1-stage tumors had depths of invasion $>5 \mathrm{~mm}$, but none of them were found to have subclinical metastases. Furthermore, in contrast to our results, Rasgon et al and Bayer et al noted that the T-stage did not correlate with cervical lymph node metastasis. ${ }^{25,26}$ This adds further contradiction to the subject; however, there still is a genuine lack of research in this particular topic.

Unfortunately, in our local population, there was limited available data regarding subclinical metastasis, especially concerning tongue carcinoma. The present study is the first of its kind to look into the chances of subclinical metastasis in early-stage diseases.

\section{Conclusion}

We conclude that in early-stage squamous cell carcinomas of the tongue (T1 and T2), a tumor thickness $>5 \mathrm{~mm}$ should be taken as an indicator for prophylactic neck dissection, while those tumors with thicknesses $\leq 5 \mathrm{~mm}$ and that have less chance of developing subclinical metastasis could be observed clinically after primary resection of the tongue lesion, thus avoiding neck dissection. This will help prevent the unnecessary morbidities of neck dissection and, at the same time, will bring less financial constraints to the patient. Poorly-differentiated tumors with a high degree of dysplasia are also one of the predictors of subclinical metastasis; but along with the exact cut-off point for the tumor thickness, it still is a debatable subject. 


\section{References}

1 Elango JK, Gangadharan P, Sumithra S, et al. Trends of head and neck cancers in urban and rural India. Asian Pac J Cancer Prev 2006;7(01):108-112

2 Goldstein DP, Irish JC. Head and neck squamous cell carcinoma in the young patient. Curr Opin Otolaryngol Head Neck Surg 2005; 13(04):207-211

3 Oc P, Pillai G, Patel S, et al. Tumour thickness predicts cervical nodal metastases and survival in early oral tongue cancer. Oral Oncol 2003;39(04):386-390

4 Kumar T, Patel M. Pattern of Lymphatic Metastasis in Relation to the Depth of Tumor in Oral Tongue Cancers: A Clinico Pathological Correlation. Indian J Otolaryngol Head Neck Surg. 2013; 65(Suppl 1):59-63

5 Okura M, Aikawa T, Sawai NY, et al. Decision analysis and treatment threshold in a management for the $\mathrm{N}_{0}$ neck of the oral cavity carcinoma. Oral Oncol 2009;45(10):908-911

6 Pentenero M, Gandolfo S, Carrozzo M. Importance of tumor thickness and depth of invasion in nodal involvement and prognosis of oral squamous cell carcinoma: a review of the literature. Head Neck 2005;27(12):1080-1091

7 Po Wing Yuen A, Lam KY, Lam LK, et al. Prognostic factors of clinically stage I and II oral tongue carcinoma-A comparative study of stage, thickness, shape, growth pattern, invasive front malignancy grading, Martinez-Gimeno score, and pathologic features. Head Neck 2002;24(06):513-520

8 Kane SV, Gupta M, Kakade AC, et al. Depth of invasion is the most significant histological predictor of subclinical cervical lymph node metastasis in early squamous carcinomas of the oral cavity. Eur J Surg Oncol 2006;32(07):795-803

9 Huang SH, Hwang D, Lockwood G, et al. Predictive value of tumor thickness for cervical lymph-node involvement in squamous cell carcinoma of the oral cavity: a meta-analysis of reported studies. Cancer 2009;115(07):1489-1497

10 Onercl M, Yilmaz T, Gedikoglu G. Tumor thickness as a predictor of cervical lymph node metastasis in squamous cell carcinoma of the lower lip. Otolaryngol Head Neck Surg 2000;122(01):139-142

11 Veness MJ, Morgan GJ, Sathiyaseelan Y, et al. Anterior tongue cancer and the incidence of cervical lymph node metastases with increasing tumour thickness: should elective treatment to the neck be standard practice in all patients? ANZ J Surg 2005;75(03):101-105

12 Jalisi S. Management of the clinically negative neck in early squamous cell carcinoma of the oral cavity. Otolaryngol Clin North Am 2005;38(01):37-46
13 Nagata M, Fujita $H$, Ida $H$, et al. Identification of potential biomarkers of lymphnode metastasis in oral squamous cell carcinoma by cDNA microarray analysis. Int J Cancer 2003; 106:683-689

14 Asakage T, Yokose T, Mukai K, et al. Tumor thickness predicts cervical metastasis in patients with stage $\mathrm{I} / \mathrm{II}$ carcinoma of the tongue. Cancer 1998;82(08):1443-1448

15 Fukano H, Matsuura H, Hasegawa Y, et al. Depth of invasion as a predictive factor for cervical lymph node metastasis in tongue carcinoma. Head Neck 1997;19(03):205-210

16 Kurokawa H, Yamashita Y, Takeda S, et al. Risk factors for late cervical lymph node metastases in patients with stage I or II carcinoma of the tongue. Head Neck 2002;24(08):731-736

17 Fakih AR, Rao RS, Borges AM, et al. Elective versus therapeutic neck dissection in early carcinoma of the oral tongue. Am J Surg 1989;158(04):309-313

18 Moore SR, Johnson NW, Pierce AM, et al. The epidemiology of tongue cancer: a review of global incidence. Oral Dis 2000; 6(02):75-84

19 Martinez-Gimeno C, Rodriguez EM, Vila CN, et al. Squamous cell carcinoma of the oral cavity: a clinicopathologic scoring system for evaluating risk of cervical lymph node metastasis. Laryngoscope 1995;105(7 Pt 1):728-733

20 Keski-Santti H, Atula T, Tornwall J, et al. Elective neck treatment versus observation in patients with T1/T2 N0 squamous cell carcinoma of oral tongue. Oral Oncol 2006;42(01):96-101

21 Pimenta Amaral TM, Da Silva Freire AR, Carvalho AL, et al. Predictive factors of occult metastasis and prognosis of clinical stages I and II squamous cell carcinoma of the tongue and floor of the mouth. Oral Oncol 2004;40(08):780-786

22 Kligerman J, Lima RA, Soares JR, et al. Supraomohyoid neck dissection in the treatment of T1/T2 squamous cell carcinoma of oral cavity. Am J Surg 1994;168(05):391-394

23 Wei WI, Ferlito A, Rinaldo A, et al. Management of the $\mathrm{N}_{0}$ neckreference or preference. Oral Oncol 2006;42(02):115-122

24 Yuen PW, Lam KY, Chan AC, et al. Clinicopathological analysis of local spread of carcinoma of the tongue. Am J Surg 1998;175(03):242-244

25 Byers RM, El-Naggar AK, Lee YY, et al. Can we detect or predict the presence of occult nodal metastases in patients with squamous carcinoma of the oral tongue? Head Neck 1998;20(02):138-144

26 Rasgon BM, Cruz RM, Hilsinger RLJr, et al. Relation of lymph-node metastasis to histopathologic appearance in oral cavity and oropharyngeal carcinoma: a case series and literature review. Laryngoscope 1989;99(11):1103-1110 